\title{
Generación de multitudes virtuales heterogéneas basadas en patrones de agrupación de comportamiento humano
}

\author{
Fernando Rebollar ${ }^{1}$, Marco A. Ramos ${ }^{1}$, Vianney Muñoz ${ }^{1}$, Félix F. Ramos ${ }^{2}$ \\ ${ }^{1}$ Universidad Autónoma del Estado de México (UAEMex), \\ Facultad de Ingeniería, México \\ ${ }^{2}$ Centro de Investigación y de Estudios Avanzados del Instituto Politécnico Nacional \\ (CINVESTAV), Unidad Guadalajara, México \\ fc@live.com.mx, marco.corchado@gmail.com, vmunozj@uaemex.mx, \\ framos@gdl.cinvestav.mx
}

\begin{abstract}
Resumen. Las ciencias computacionales en conjunto con otras áreas del conocimiento participan en el estudio del comportamiento de masas de individuos, intentando predecir y anticipar situaciones que puedan presentarse, a través de simulaciones de multitudes que permitan una experimentación a bajo costo y que aporten datos a las organizaciones para predecir y anticipar distintas eventualidades. Para ello, es necesario realizar simulaciones con multitudes virtuales que se comporten lo más parecido a la realidad. En este artículo se presenta una forma de generar multitudes virtuales con comportamientos heterogéneos, de tal forma que los individuos que conforman la multitud tengan distintos comportamientos. Se propone una técnica de agrupamiento en distintas regiones del área a poblar utilizando diagramas de Voronoi, posibilitando la caracterización de zonas donde existe una concentración masiva de personas en un espacio específico como lo son: plazas, centros comerciales, aeropuertos, escuelas, etc.
\end{abstract}

Palabras clave: Inteligencia artificial, agentes, multitudes heterogéneas.

\section{Generation of Heterogeneous Virtual Crowds based on Patterns of Grouping of Human Behavior}

Resumen. Computer science with other areas of knowledge is involved in the study of the individual's behavior of the crowd, trying to predict and anticipate situations that may arise. In crowd simulations, we can see differents experimentations that provide the different behavior on specific conditions or eventualities not controlled. In this paper, we present one technique based on Voronoi diagrams to defined the local concentration of people (LCP) that permitted create the heterogeneous crowd. LCP 
identified the principal place of environment where to exist the possibility of a crowd. The Voronoi diagrams help to agents to find the particular location to access a resource to complete the main goal. The first results showed the way of how the agents can crowd like the circles, delta, and oval groupping.

Keywords. Artificial intelligence, agents, heterogeneous crowds.

\section{Introducción}

El estudio de la concentración de individuos en lugares públicos como plazas, centros comerciales, parques, jardines, etc., es un campo de estudio abierto en las diferentes disciplinas de las ciencias. Conllevando a la necesidad de disponer de sistemas que permitan pronosticar y predecir eventualidades en situaciones no controladas, como es en el caso de un terremoto. De ahí que, las ciencias computacionales como la inteligencia artificial investigan cómo replicar el comportamiento humano virtualmente, para obtener simulaciones que sean lo más apegado a la realidad, apoyándose de las áreas sociales, psicológicas, neurocientíficas, entre otras, con la finalidad de empatar estas teorías en el área de la inteligencia artificial.

El principal propósito de estudiar y simular multitudes virtuales es representar con precisión grupos de individuos autónomos llamados agentes virtuales que atienden a las mismas reglas en entornos cercanos a aquellos encontrados en la vida real. Para anticipar contingencias y atenderlas adecuadamente antes de que sucedan es necesario estudiar el comportamiento de las masas de individuos en actividades cotidianas, permitiendo la construcción de la infraestructura requerida de acuerdo a las necesidades de los individuos que hacen uso de ella, por ejemplo, las plazas, los aeropuertos, las escuelas, el transporte público, etc. A esta infraestructura se le conoce como ciudades inteligentes.

Grupos de investigación alrededor del mundo se han sumado al problema de la simulación de multitudes virtuales con grandes logros desde el realismo obtenido hasta el comportamiento embebido en los agentes virtuales. Sin embargo, sus simulaciones carecen de heterogeneidad en la población ya que sus estudios se enfocan en un sólo modelo que replican infinitamente en el medio ambiente virtual, esto resulta menos costoso computacionalmente comparado a la generación diversos modelos de acuerdo a la heterogeneidad de la población e implica que el comportamiento observado dentro de la simulación sea idéntico para todos los agentes virtuales [4].

En la realidad no existe un número determinado de individuos que constituyen una multitud, no obstante, el estudio de las multitudes se basa en el mayor número de individuos concentrados en un espacio observando su comportamiento en situaciones de estrés, manteniendo la meta de poder anticipar contingencias en tiempo real [11]. Las aproximaciones computacionales para la generación de multitudes son centradas en las reglas propias del ambiente físico y el comportamiento está basado en la interacción del ambiente y las reglas físicas del mismo. 
Generación de multitudes virtuales heterogéneas basadas en patrones de agrupación ...

Uno de los problemas que se tienen con la generación de multitudes es la diversidad visual para representar la heterogeneidad en las multitudes. Así como la planificación que deberán de realizar los agentes dentro del ambiente virtual para el logro de sus objetivos [22].

Para reproducir comportamientos individuales y grupales de forma exitosa dos problemas fundamentales deben abordarse: la planificación y la toma de decisiones. La planificación dotará a los agentes para poder observar el medio ambiente y poder decidir sobre las condiciones del mismo, por ejemplo la evasión de colisiones. Los métodos principales para la evasión de colisiones se basan en fuerzas sociales [9] mientras que la velocidad es manejada de manera recíproca para todos los agentes. Un algoritmo simple para evitar colisiones compara la posición de cada agente con los demás, sin embargo conforme el número de agentes (denotado por $N$ ) crece, la complejidad del algoritmo es $O\left(N^{2}\right)$. Dicha complejidad representa un problema cuando se requiere simular grandes multitudes e imposibilita la obtención de una simulación en tiempo real. Para que una simulación de multitudes sea precisa es necesario que reproduzca comportamientos humanos individuales y grupales, además los algoritmos que sintetizan estos comportamientos deben estar optimizados para trabajar en tiempo real [17].

El comportamiento colectivo en los humanos se ha estudiado desde principios del siglo XIX con la finalidad de observar las acciones que las personas realizan cuando se presentan diferentes circunstancias como celebraciones, manifestaciones, simulacros o incluso cuando ocurren fenómenos naturales como incendios o terremotos [6]. Las ciencias computacionales realizan esfuerzos para simular de manera virtual multitudes con el objetivo de reproducir comportamientos similares a los de los seres humanos con la finalidad de estudiar eventualidades en las multitudes [4].

Las simulaciones de multitudes son realizadas a partir de diferentes enfoques dependiendo del ambiente virtual que se desea poblar, por ejemplo: la industria del entretenimiento (videojuegos, películas, realidad virtual, etc.). Este tipo de simulaciones ha tomado importancia en el uso de los llamados juegos serios [16]. Estos últimos requieren ser poblados con agentes que permitan al usuario contar con una retroalimentación o ser asistido en tareas inmersas en el ambiente virtual.

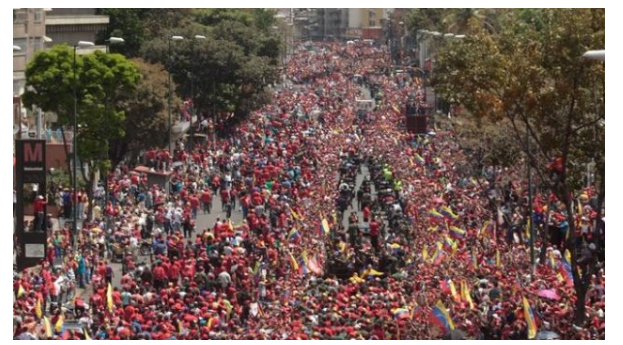

Fig. 1. Multitud de personas en avenidas públicas. 
Fernando Rebollar Castelan, Marco A. Ramos Corchado, Vianney Muñoz Jiménez, et al.

Este tipo de simulaciones permite a las diferentes organizaciones garantizar la integridad de los individuos aglomerados en espacios públicos, como se ilustra en la Figura 1. Las organizaciones hacen uso de las simulaciones basadas en esté tipo con el propósito de prevenir situaciones no controladas en el entorno [25].

\section{Trabajos relacionados}

El estudio de las multitudes es de suma importancia y se puede observar ante la presencia de fenómenos naturales, por ejemplo los cardumen de sardinas, en donde las especies jóvenes se encuentran en el centro para protegerse de los predadores y preservar la especie. En 1987 Craig Reynolds presentó uno de los primeros trabajos sobre la creación de multitudes basados en el comportamiento de las aves y la manera de cómo se agrupan en vuelo. Las investigaciones de Reynolds encaminan a la generación de multitudes basadas en humanoides siguiendo tres reglas simples: separación, alineación y cohesión. Según Reynolds un ave es consciente de tres elementos durante el vuelo: conocimiento de sí mismo, vecinos cercanos, y un líder a seguir [15].

Los trabajos de Reynolds funcionan en agentes que tienen un mismo comportamiento como son las aves y otro tipo de animales. Sin embargo, las multitudes basadas en humanos es más compleja debido a la personalidad de cada uno de los individuos que participan en una multitud, donde diversos comportamientos deben de ser considerados para que la simulación se apegue más a la realidad.

Es importante considerar en una simulación de multitud un comportamiento reactivo, es decir asociar todas aquellas acciones que son desencadenadas mediante un evento, en tanto no se registre ningún estimulo del ambiente o incluso de algún otro agente involucrado en la simulación. Los agentes que pertenezcan a la categoría reactiva no tienen ninguna razón para reaccionar [4]. Erik Millan genera máquinas de estado finitas desde archivos XML y las guarda en imágenes que los agentes pueden consultar [11]. Pelechano y Badler han combinado reglas de percepción y comportamientos reactivos para dirigir agentes en entornos virtuales [13]. Sí los comportamientos reactivos son implementados correctamente pueden tener como consecuencia comportamientos emergentes que producen simulaciones más realistas.

Las simulaciones basadas en reglas no requieren de un razonamiento complejo, esto es debido a que el medio ambiente es el responsable de seleccionar la mejor acción que los agentes deben seguir en las diferentes situaciones que se les presentan [20]. Sin embargo, esto se aleja a lo que sucede en un entorno real porque el ambiente no controla las acciones de los peatones, sólo los limita. Kapadia [10], combinó predicciones de espacio-tiempo, comportamientos reactivos, y movimientos de dirección en plataformas dedicadas a la simulación de multitudes, lo que da libertad a los agentes de tomar sus decisiones de desplazamiento por ellos mismos.

Lograr simulaciones de multitudes lo más parecidas a la realidad, requiere de resolver el problema de coaliciones que medido computacionalmente es de $O\left(N^{2}\right)$, donde $N$ es el número de agentes que participaran en la simulación [17]. 
Generación de multitudes virtuales heterogéneas basadas en patrones de agrupación ...

Esto significa que cada agente tiene que consultar a todos los otros agentes por su posición y otra información importante que le permita calcular su dirección y velocidad con respecto a la de sus vecinos. Esto reduce la complejidad de las búsquedas de proximidad, permitiendo simulaciones de grandes multitudes.

Para reducir la complejidad en el paso de mensajes entre los agentes se utilizan estructuras jerárquicas, como los octrees, en donde el espacio se subdivide en varias regiones que contienen agentes donde los integrantes de una región conocen solo a los agentes que estén dentro de la misma región [8]. Mejoras a la estructura octrees, utilizan árboles $k d$ para que al consultar a los vecinos más cercanos sea un proceso eficiente. Bleiweiss en [1] presentó una implementación en paralelo de la biblioteca popular para evasión de colisiones, obteniendo un aumento de velocidad de 4.8X en comparación con la implementación original. Bleiweiss cambio el método de búsquedas de proximidad de un árboles $k d$ a un método basado en tablas hash con el fin de mejorar el rendimiento en la Unidad de Procesamiento Gráfico (GPU).

En 2004, Chenney [2] propone una técnica para la representación y el diseño de campos de velocidad, usando autómatas celulares útiles para crear movimientos de flujo, que son seguidos por los peatones, con el fin de moverse a través de un entorno, ver la Figura 2. En 2011, Zhang [24] presenta un modelo en el que las celdas de un autómata celular representan posiciones discretas en el espacio, utilizadas por los peatones para moverse al cambiar de una celda a otra generando simulaciones más realistas.

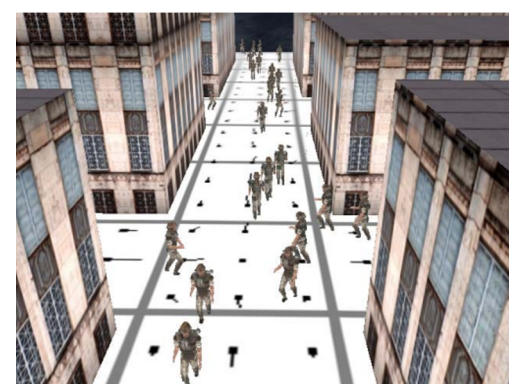

Fig. 2. Simulación de Chenney usando autómatas celulares [2].

Guy en 2010, presenta un método que calcula trayectorias reduciendo al mínimo el esfuerzo que los agentes necesitan llevar a cabo para llegar a su destino [7]. El algoritmo es capaz de evitar colisiones con otros agentes y los obstáculos, al tiempo que permite simulaciones en tiempo real [12].

Van den Berg mejora el algoritmo de RVO (Velocidades Reciprocas para Obstáculos) reduciendo el problema a un programa lineal de baja dimensión [23], y con ello son capaces de simular multitudes de miles de personajes. En la Figura 3 se observa una prueba con 1,000 agentes en tiempo real. 
Fernando Rebollar Castelan, Marco A. Ramos Corchado, Vianney Muñoz Jiménez, et al.

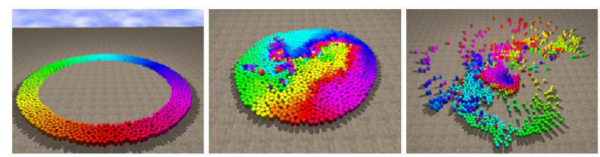

Fig. 3. Los agentes se mueven hacia la posición opuesta de donde comienzan en el círculo.

D. Thalmann [21], remarca que las principales razones que dificultan el uso de multitudes virtuales es precisamente el dominio de tiempo real y las altas exigencias al CPU, así como los altos costos de la producción de contenidos. Thalmann propone algoritmos para optimizar el hardware haciendo posible mostrar escenas virtuales en $3 \mathrm{D}$ con miles de entidades individuales animadas que anteriormente no era posible, su simulador permite crear miles de agentes donde la multitud se mueve de un lugar a otro en tiempo real (ver Figura 4).

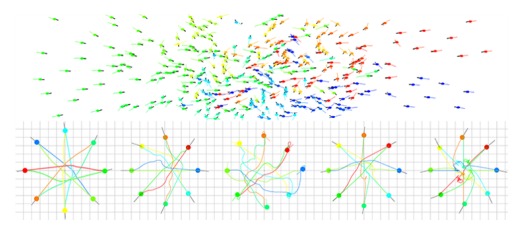

Fig. 4. Simulación en tiempo real de Thalmann [14].

\section{Generación de comportamientos heterogéneos}

Un problema a abordar en la generación de multitudes es la carencia de diversidad que la conforman, debido a que sólo se utiliza un modelo del agente que se replica de manera discriminada, cambiando sólo el color de la ropa o cabello. También podemos observar la falta de autonomía en los agentes provocando un solo comportamiento lo cual reduce el realismo en la simulación.

Nuestra propuesta se basa en la generación de multitudes heterogéneas a través de diversos modelos que conforman la multitud sin descuidar los comportamientos asociados a cada agente. Por ejemplo, sí en la escena se observa un anciano este debe tener el comportamiento de un anciano.

\subsection{El comportamiento humano}

El comportamiento de cada persona en la vida real es único, el cual esta determinado por un sin fin de factores que lo van determinando a lo largo del tiempo y de experiencias vividas por cada persona. También lo delimitan aspectos fisiológicos y capacidades que impiden poder realizar algunas actividades para determinados sectores de la población. 
Generación de multitudes virtuales heterogéneas basadas en patrones de agrupación ...

Lo ideal en las simulaciones de multitudes es que cada agente se comportara de manera diferente tal y como pasa en la vida real. Sin embargo, este proceso tomaría demasiados recursos del sistema para que cada agente virtual contara con un comportamiento individual impidiendo su simulación en tiempo real.

Samson en [19] determinó la velocidad de desplazamiento, cadencia de los pasos y longitud de zancada de las personas, considerando parámetros como la edad, el peso y la altura de las personas. Para ello, analizó a 118 mujeres y 121 hombres en un rango de entre los 19 a 90 años de edad, los cuales caminaban a su velocidad preferida como lo harían normalmente a través de una pasarela de 12 metros. Samson propone formulas para calcular la velocidad de desplazamiento de los individuos en actividades cotidianas. En nuestro caso de estudio retomamos las ecuaciones propuestas por Samson para que los agentes virtuales reproduzcan estos desplazamientos con la característica que podemos distinguir hombres y mujeres dentro de la simulación. La Tabla 1 muestra la relación de velocidad desplazamiento existente entre hombres y mujeres.

Tabla 1. Ecuaciones de velocidades de desplazamiento en metros/segundos de los seres humanos de Samson [19].

\begin{tabular}{|l|}
\hline Hombres \\
\hline Velocidad $=1.460$ \\
Velocidad $=-0.002$ edad $\left(^{*}\right)+1.582$ \\
Velocidad $=-0.002$ edad $\left(^{*}\right)+0.442$ altura $(*)+0.750$ \\
Velocidad $=-0.001$ edad $\left(^{*}\right)+0.486$ altura $(*)-0.001$ peso $\left(^{*}\right)+0.720$ \\
\hline Mujeres \\
\hline Velocidad $=1.420$ \\
Velocidad $=-0.003$ edad $(*)+1.552$ \\
Velocidad $=-0.002$ edad $\left(^{*}\right)+0.618$ altura $(*)+0.484$ \\
Velocidad $=-0.001$ edad $(*)+0.827$ altura $(*)-0.003$ peso $\left(^{*}\right)+0.316$ \\
\hline
\end{tabular}

La posibilidad de dotar a los agentes de valores aleatorios en el desplazamiento, así como condicionantes de masa y altura permite obtener una simulación dentro de la multitud lo más cercana a la realidad. Los factores asociados a la física del ambiente son comportamientos emergentes de acuerdo a las diferentes masas de los agentes que participan en la simulación. Los agentes están provistos de sensores que les permiten recalcular sus trayectorias para evitar colisionar con objetos dentro de ambiente y con otros agentes. En un sistema multiagentes el ambiente se define como el conjunto $E=\left\{e, e^{\prime}, \ldots\right\}$ donde $E$ es el ambiente conformado por todos los posibles estados. $A c=\left\{\alpha, \alpha^{\prime}, \ldots\right\}$ representa todas las acciones permitidas dentro de $E$. Los agentes construyen su base de conocimientos a partir de $r: e_{0} \stackrel{\alpha_{0}}{\rightarrow} e_{1} \stackrel{\alpha_{1}}{\rightarrow} e_{2} \stackrel{\alpha_{2}}{\rightarrow} e_{3} \stackrel{\alpha_{3}}{\rightarrow} \ldots \stackrel{\alpha_{u}-1}{\rightarrow} e_{u}$ que representan las acciones realizadas de un estado a otro dentro de $E$. 


\subsection{Distribución geométrica espacial del ambiente}

La geografía del ambiente es un factor importante en la generación de multitudes, los agentes virtuales que ocuparán el espacio deberán contar con información inicial que les permita reconocer su entorno y poder lograr sus objetivos, así mismo saber qué lugares son los que pueden visitar y los que deben de evitar. Para resolver esto, en nuestro caso hacemos uso de los diagramas de Voronoi que permiten dividir un área en regiones bien definidas.

Sea $P=\left\{p_{1}, p_{2}, \ldots, p_{n}\right\}$ un conjunto de puntos en el plano, haciendo uso del diagrama de Voronoi es posible asignar a cada punto una región en el plano correspondiente a los puntos más cercanos, una región para cada $p_{i} \in P$, todos los puntos asignados a $p_{i}$ en el conjunto de puntos $P$ forman la región de Voronoi $V\left(p_{i}\right)$ [3]. Dado un conjunto de puntos en $P$ y un punto de consulta $q$, es posible determinar el punto más cercano a $q$ en $P$, dado que la ubicación de $q$ esta dentro de una región de Voronoi en un punto $p_{i}$ la cual indica que dicho punto $p_{i}$ es el más cercano al punto $q$.

$$
V\left(p_{i}\right)=q\left|\left\|p_{i} q\right\|\right|<\left\|p_{j} q\right\|, \forall j \neq i,
$$

donde $\|p q\|$ es la distancia euclídea entre $p$ y $q$.

Como primer aproximación podemos decir que dado un conjunto $P$ de sitios (puntos) en el plano, su diagrama de Voronoi es la partición de ese plano en regiones (una región para cada sitio), tal que la región del sitio $p$ contiene todos los puntos del plano que están más cerca de $p$ que de cualquier otro sitio en $P$.

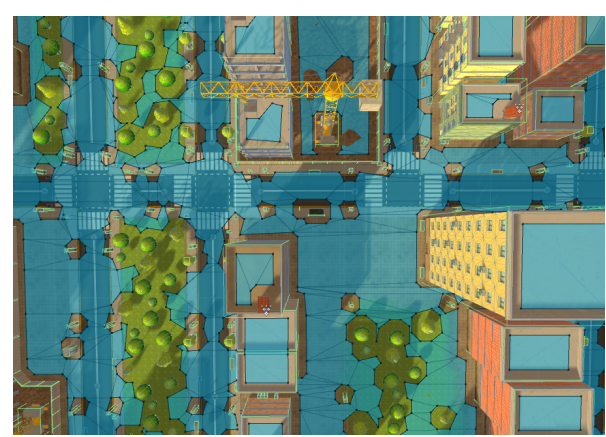

Fig. 5. Ubicación de LCP dentro del ambiente.

Nuestro caso de estudio hace uso del algoritmo Steven Fortune para generar el diagrama de Voronoi ya que dicho algoritmo [5] se ejecuta en $O(n \log n)$ por lo que se puede notar que es un algoritmo en $O(n)$ considerado uno de los mejores actualmente. Una vez generado el diagrama de Voronoi es utilizado para determinar la correcta distribución de la población dentro de ambiente virtual, para los primeros resultados trabajaremos con una ciudadela simulada 
Generación de multitudes virtuales heterogéneas basadas en patrones de agrupación ...

(ver Figura 5), donde los puntos $p$ generados por Voronoi los llamaremos lugares de concentración de población (LCP). Un LCP puede ser una parada de autobús, una tienda comercial, etc. El uso de LCP nos permite conceptualizar los espacios dentro del ambiente y poder definir la concentración de individuos por regiones, por ejemplo si la región esta marcada como escuela primaria, dicha región deberá ser poblada en su mayoría de agentes que representen niños y niñas en un rango de edad entre los 6 y 12 años.

La posibilidad de contar con varios LCP nos permitirá poblar ambientes mucho más complejos y así poder observar y estudiar los comportamientos asociados y emergentes dentro de una población completamente heterogénea.

\section{Resultados}

El software utilizado para las simulaciones de los agentes fue Unity 5.3 de 64 bits, en una computadora con procesador intel i7 de $3.4 \mathrm{GHz}$ y 4 GB en RAM. Los primeros resultados obtenidos se basan en los desplazamientos que toman los agentes dentro del ambiente virtual en donde es posible distinguir desplazamientos heterogéneos, inicialmente los agentes están representados por cubos de diferentes tamaños y masas para dotarlos de características individuales.

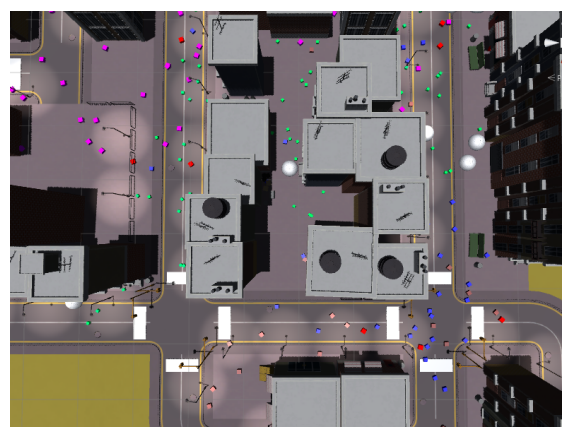

Fig. 6. Agentes virtuales, desplazándoce por la ciudad buscando su LCP de interés.

Los agentes son identificados por colores para clasificarlos en 5 tipos de roles dentro del ambiente. Los de color verde representan a la población de niños entre 4 a 14 años de edad, los de color azul representan a jóvenes de 15 a 24 años, los rojos representan a adultos de 25 a 54 años de edad, los violeta representan a adultos mayores de 55 a 64 años y por último los de color café representan a los ancianos de más de 65 años. La Figura 6 muestra la representación de los agentes poblando el medio ambiente virtual.

La implementación de las ecuaciones de velocidad propuestas por Samson permiten observar desplazamientos de tipo natural. Además de la creación de los LCP, los agentes tienen la posibilidad de tomar diferentes caminos ocupando todo 
el espacio geográfico del ambiente permitiendo lograr comportamientos parecidos a los reales.

En la Figura 6 se muestra la simulación de los agentes desplazándose por las calles de la ciudad, rumbo a su LCP objetivo, se observa como los agentes toman en cuenta al resto de los agentes dentro del ambiente evitando colisionar con otros. Se puede ver así mismo como se comienza acumular una masa importante de agentes intentado acceder al recurso, lo que produce saturación en determinadas zonas. Los LCP permiten concentrar agentes de un mismo tipo, sin embargo es válido encontrar agentes de otro tipo en la misma zona, esto es debido a las rutas que toman los agentes y son comportamientos similares a los vistos en la realidad. Finalmente la emergencia de comportamientos como la agrupación de multitudes resulta de la necesidad de acceder a un recurso, tal como sucede en la realidad.

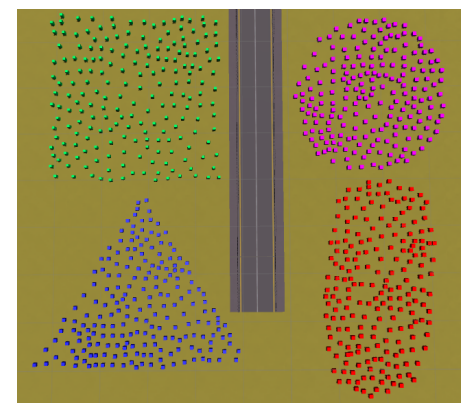

Fig. 7. Patrones de multitudes obtenidas por los agentes.

Uno de los aspectos importantes de las multitudes es la forma en cómo estas se agrupan [18]. Los expertos en comportamiento humano han determinado ciertos patrones en los que destacan la agrupación cuadrada, circular, delta y ovoide. El siguiente resultado se obtuvo al forzar a los agentes a agruparse en estos patrones, sin que exista una orden directa, esto lo hacen de acuerdo a la negociación de ocupar un espacio, sabiendo que la tarea es completa en el momento en que todos los agentes logren el patrón. La Figura 7 muestra los patrones obtenidos por los agentes, es importante resaltar que la distancia entre agentes no está dada, en caso contrario el resultado final sería una formación tipo militar (alineada), lo que no sucede en el comportamiento de una población real de civiles.

En muchas de las manifestaciones que involucran multitudes estas se agrupan en pequeños grupos y se van uniendo a otros para llegar al objetivo final. Estos pequeños grupos deben sortear diversos obstáculos para alcanzar al contingente mayor, en nuestra siguiente prueba a manera de experimentación lo que hacemos es integrar dos grupos de multitudes con diferente patrón y observar como se realiza la integración en un solo grupo y que patrón se obtiene como resultado. Esté comportamiento se observa en la Figura 8, en el momento en que comienzan el desplazamiento estos rompen el patrón inicial y una vez que se forma el 
Generación de multitudes virtuales heterogéneas basadas en patrones de agrupación ...

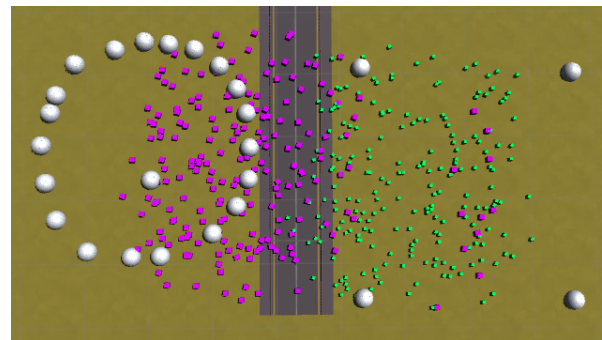

Fig. 8. Patrones de multitudes obtenidas por los agentes.

nuevo grupo se mantiene un patrón de forma cuadrada cuando se trata de desplazamiento y de forma circular u ovoide en el caso de espera. El patrón delta se forma en presencia de un agente líder a seguir.

En el vídeo ${ }^{1}$ en la web se pueden observar dos experimentaciones en donde los agentes se desplazan a sus objetivos negociando espacios y calculando sus rutas, también en el mismo vídeo se puede observar la integración de las dos multitudes que se muestran en la Figura 8.

\section{Conclusiones}

El uso de las velocidades de desplazamiento de los seres humanos utilizado en las simulaciones de multitudes, permite observar comportamientos parecidos a los reales. La categorización de los individuos por edades genera comportamientos indistintos, lo que permite observar en la simulación comportamientos lo más parecidos a la realidad. Una de las aportaciones que se realizan en este trabajo es la creación de LCPs utilizando diagrama de Voronoi lo que nos permite generar concentraciones de individuos en un espacio al cual los agentes necesitan acceder como recurso, además de ver como los agentes realizan procesos de comunicación y negociación para el logro de sus objetivos individuales. La identificación de LCPs permite observar concentración heterogénea de agentes de acuerdo a un contexto, por ejemplo un parque deberá ser poblado en su mayoría por ancianos, niños y mujeres. Poder reproducir los patrones de las agrupaciones de las multitudes con los agentes virtuales nos permite estudiar los comportamientos como suceden en la vida real, permitiendo a las organizaciones evaluar las condiciones e implementar las políticas de posibles contingencias.

\section{References}

1. Bleiweiss, A.: Multi agent navigation on the gpu. In: GDC09 Game Developers Conference. vol. 2009 (2009)

${ }^{1}$ https://youtu.be/GaEpYtvak04 
Fernando Rebollar Castelan, Marco A. Ramos Corchado, Vianney Muñoz Jiménez, et al.

2. Chenney, S.: Flow tiles. In Proceedings of the 2004 ACM SIGGRAPH/Eurographics symposium on Computer animation - SCA pp. 233-242 (2004)

3. De Berg, M., Van Kreveld, M., Overmars, M., Schwarzkopf, O.C.: Computational geometry. In: Computational geometry, pp. 147-169. Springer (2000)

4. De Gyves, O., Toledo, L., Rudomin, I.: Comportamientos en simulación de multitudes: revisión del estado del arte. Research in Computer Science pp. 319-334 (2013)

5. Fortune, S.: A sweepline algorithm for voronoi diagrams. Algorithmica 2(1-4), 153$174(1987)$

6. G, L.: Psychologie des foules. Alcan, Paris (1895)

7. Guy, S.J., Chhugani, J., Curtis, S., Dubey, P., Lin, M., Manocha, D.: Pledestrians: a least-effort approach to crowd simulation. In: Proceedings of the 2010 ACM SIGGRAPH/Eurographics symposium on computer animation. pp. 119-128. Eurographics Association (2010)

8. Hadap, S., Eberle, D., Volino, P., Lin, M.C., Redon, S., Ericson, C.: Collision detection and proximity queries. In: ACM SIGGRAPH 2004 Course Notes. p. 15. ACM (2004)

9. Helbing, D., Molnar, P.: Social force model for pedestrian dynamics. Physical review E 51(5), 4282 (1995)

10. Kapadia, M., Singh, S., Reinman, G., Faloutsos, P.: A behavior-authoring framework for multiactor simulations. Computer Graphics and Applications, IEEE 31(6), 45-55 (2011)

11. Millan, E., Hernández, B., Rudomin, I.: Large crowds of autonomous animated characters using fragment shaders and level of detail. ShaderX5: Advanced Rendering Techniques pp. 501-510 (2007)

12. Paolo Fiorini, Z.S.: Motion planning in dynamic environments using velocity obstacles. The International Journal of Robotics Research pp. 760-772 (July 1998)

13. Pelechano, N., Allbeck, J.M., Badler, N.I.: Controlling individual agents in high-density crowd simulation. In: Proceedings of the 2007 ACM SIGGRAPH/Eurographics symposium on Computer animation. pp. 99-108. Eurographics Association (2007)

14. Pettré, J., Ciechomski, P.d.H., Maïm, J., Yersin, B., Laumond, J.P., Thalmann, D.: Real-time navigating crowds: scalable simulation and rendering. Computer Animation and Virtual Worlds 17(3-4), 445-455 (2006)

15. Reynolds, C.W.: Flocks, herds and schools: A distributed behavioral model. ACM SIGGRAPH Computer Graphics (August 1987)

16. Ritterfeld, U., Cody, M., Vorderer, P.: Serious games: Mechanisms and effects. Routledge (2009)

17. Ruiz, S., Hernández, B.: Procesos de decisión de markov y microescenarios para navegación y evasión de colisiones para multitudes. ResearchGate (2014)

18. S. Raupp Musse, D.: A behavioral model for real time simulation of virtual human crowds. IEEE Transactions on Visualization and Computer Graphics 7(2), 152-164 (2001)

19. Samson, M., Crowe, A., De Vreede, P., Dessens, J., Duursma, S., Verhaar, H.: Differences in gait parameters at a preferred walking speed in healthy subjects due to age, height and body weight. Aging Clinical and Experimental Research 13(1), 16-21 (2001)

20. Sun, L., Qin, W.: Simulation of crowd behaviors based on event reaction. In: Computer Science and Automation Engineering (CSAE), 2011 IEEE International Conference on. vol. 2, pp. 163-167. IEEE (2011) 
Generación de multitudes virtuales heterogéneas basadas en patrones de agrupación ...

21. Thalmann, D.: Populating virtual environments with crowds. In: Proceedings of the 2006 ACM international conference on Virtual reality continuum and its applications. ACM (2006)

22. Thalmann, D., Grillon, H., Maim, J., Yersin, B.: Challenges in crowd simulation. In: 2009 International Conference on CyberWorlds. pp. 1-12. IEEE (2009)

23. Van Den Berg, J., Guy, S.J., Lin, M., Manocha, D.: Reciprocal n-body collision avoidance. In: Robotics research, pp. 3-19. Springer (2011)

24. Zhang, S., Li, M., Li, F., Liu, A., Cai, D.: A simulation model of pedestrian flow based on geographical cellular automata. In: Geoinformatics, 2011 19th International Conference on. pp. 1-5. IEEE (2011)

25. Zhong, Z., Ding, N., Wu, X., Xu, Y.: Crowd surveillance using markov random fields. In: Automation and Logistics, 2008. ICAL 2008. IEEE International Conference on. pp. 1822-1828. IEEE (2008) 